\title{
Sandpiles and Dominos
}

\author{
Laura Florescu \\ Courant Institute \\ New York University \\ New York, U.S.A. \\ florescu@cims.nyu.edu
}

\author{
Daniela Morar \\ Department of Economics \\ University of Michigan \\ Ann Arbor, U.S.A. \\ morard@umich.edu
}

\author{
David Perkinson \\ Department of Mathematics \\ Reed College \\ Portland, U.S.A. \\ davidp@reed.edu
}

\author{
Nick Salter \\ Department of Mathematics \\ University of Chicago \\ Chicago, U.S.A. \\ nks@math.uchicago.edu
}

\author{
Tianyuan $\mathrm{Xu}$ \\ Department of Mathematics \\ University of Oregon \\ Eugene, U.S.A. \\ tianyuan@uoregon.edu
}

Submitted: Jun 18, 2014; Accepted: Feb 24, 2015; Published: Mar 13, 2015

Mathematics Subject Classifications: 05C57

\begin{abstract}
We consider the subgroup of the abelian sandpile group of the grid graph consisting of configurations of sand that are symmetric with respect to central vertical and horizontal axes. We show that the size of this group is (i) the number of domino tilings of a corresponding weighted rectangular checkerboard; (ii) a product of special values of Chebyshev polynomials; and (iii) a double-product whose factors are sums of squares of values of trigonometric functions. We provide a new derivation of the formula due to Kasteleyn and to Temperley and Fisher for counting the number of domino tilings of a $2 m \times 2 n$ rectangular checkerboard and a new way of counting the number of domino tilings of a $2 m \times 2 n$ checkerboard on a Möbius strip.
\end{abstract}

\section{Introduction}

This paper relates the Abelian Sandpile Model (ASM) on a grid graph to domino tilings of checkerboards. The ASM is a chip-firing game encoding the dynamics implicit in the discrete Laplacian of a graph. It arises in statistical physics ([1], [9], [10], [14], [13], [41]), algebraic geometry and commutative algebra ([2], [11], [7], [27], [26], [39]), pattern formation ([34], [36], [38], [37], [42]), potential theory ([3], [5], [23]), combinatorics ([4], [8], [17], [18], [22], [24], [30], [40]), and number theory ([32]). The citations here are by no means exhaustive. A good general reference for our purposes is [16]. In the ASM, one imagines placing grains of sand on the vertices of a graph. If the amount of sand on a vertex reaches a certain threshold, the configuration of sand becomes unstable. 
There is a stabilization process in which vertices fire sand to their neighbors and excess sand is eventually absorbed by a specified sink vertex. The model guarantees that each configuration of sand has a unique stabilization. A special subcollection of the set of stable configurations of sand are the so-called recurrent configurations. These form a group under vertex-wise addition of grains of sand followed by stabilization. This group is called the sandpile group or critical group of the graph.

At least since the seminal paper by Bak, Tang, and Wiesenfeld (BTW), [1], there has been special interest in the ASM on the sandpile grid graph. To construct the $m \times n$ sandpile grid graph, start with the ordinary grid graph with vertices $[m] \times[n]$ and edges $\left\{(i, j),\left(i^{\prime}, j^{\prime}\right)\right\}$ such that $\left|i-i^{\prime}\right|+\left|j-j^{\prime}\right|=1$. Then add a new vertex to serve as a sink, and add edges from the boundary vertices to the sink so that each vertex on the grid has degree 4. Thus, corner vertices have two edges to the sink (assuming $m$ and $n$ are greater than 1), as on the left in Figure 6.

The initial motivation for our work was a question posed to the second and third authors by Irena Swanson ${ }^{1}$. She was looking at an online computer program [28] for visualizing the ASM on a sandpile grid graph. By pushing a button, the program adds one grain of sand to each of the nonsink vertices then stabilizes the resulting configuration. Swanson asked, "Starting with no sand, how many times would I need to push this button to get the identity of the sandpile group?" A technicality arises here: the configuration consisting of one grain of sand on each vertex is not recurrent, hence, not in the group. However, the all-2s configuration, having two grains at each vertex, is recurrent. So for the sake of this introduction, we reword the question as: "What is the order of the all-2s configuration?"

Looking at data (cf. Section 5, Table 1), one is naturally led to the special case of the all-2s configuration on the $2 n \times 2 n$ sandpile grid graph, which we denote by $\overrightarrow{2}_{2 n \times 2 n}$. The orders for $\overrightarrow{2}_{2 n \times 2 n}$ for $n=1, \ldots, 5$ are

$$
1,3,29,901,89893 .
$$

Plugging these numbers into the Online Encyclopedia of Integer Sequences yields a single match, sequence A065072 ([33]): the sequence of odd integers $\left(a_{n}\right)_{n \geqslant 1}$ such that $2^{n} a_{n}^{2}$ is the number of domino tilings of the $2 n \times 2 n$ checkerboard. ${ }^{2}$ (Some background on this sequence is included in Section 5.) So we conjectured that the order of $\overrightarrow{2}_{2 n \times 2 n}$ is equal to $a_{n}$, and trying to prove this is what first led to the ideas presented here. Difficulty in finishing our proof of the conjecture led to further computation, at which time we (embarrassingly) found that the order of $\overrightarrow{2}_{2 n \times 2 n}$ for $n=6$ is, actually, $5758715=a_{6} / 5$. Thus, the conjecture is false, and there are apparently at least two natural sequences that start $1,3,29,901,89893$ ! Theorem 29 shows that the cyclic group generated by $\overrightarrow{2}_{2 n \times 2 n}$ is isomorphic to a subgroup of a sandpile group whose order is $a_{n}$, and therefore the order

\footnotetext{
${ }^{1}$ Oral communication.

${ }^{2}$ By a checkerboard we mean a rectangular array of squares. A domino is a $1 \times 2$ or $2 \times 1$ array of squares. A domino tiling of a checkerboard consists of covering all of the squares of the checkerboard-each domino covers two - with dominos.
} 
of $\overrightarrow{2}_{2 n \times 2 n}$ divides $a_{n}$. We do not know when equality holds, and we have not yet answered Irena Swanson's question.

On the other hand, further experimentation using the mathematical software Sage led us to a more fundamental connection between the sandpile group and domino tilings of the grid graph. The connection is due to a property that is a notable feature of the elements of the subgroup generated by the all-2s configuration-symmetry with respect to the central horizontal and vertical axes. The recurrent identity element for the sandpile grid graph, as exhibited in Figure 1, also has this symmetry. ${ }^{3}$



Figure 1: Identity element for the sandpile group of the $400 \times 400$ sandpile grid graph.

If $\Gamma$ is any graph equipped with an action of a finite group $G$, it is natural to consider the collection of $G$-invariant configurations. Proposition 6 establishes that the symmetric recurrent configurations form a subgroup of the sandpile group for $\Gamma$. The central purpose of this paper is to explain how symmetry links the sandpile group of the grid graph to domino tilings.

We now describe our main results. We study the recurrent configurations on the sandpile grid graph having $\mathbb{Z} / 2 \times \mathbb{Z} / 2$ symmetry with respect to the central horizontal and vertical axes. The cases of even $\times$ even-, even $\times$ odd-, and odd $\times$ odd-dimensional grids each have their own particularities, and so we divide their analysis into separate cases, resulting in Theorems 15, 18, and 23, respectively. In each case, we compute the number of symmetric recurrents as (i) the number of domino tilings of corresponding (weighted) rectangular checkerboards; (ii) a product of special values of Chebyshev polynomials; and (iii) a double-product whose factors are sums of squares of values of trigonometric functions.

\footnotetext{
${ }^{3}$ For square grids, the identity is symmetric with respect to the dihedral group of order 8 , but this phenomenon is of course not present in the rectangular grids that we also consider.
} 
For instance, of the 557, 568, 000 elements of the sandpile group of the $4 \times 4$ grid graph, only the 36 configurations displayed in Figure 8 are up-down and left-right symmetric. In accordance with Theorem 15,

$$
\begin{aligned}
36 & =U_{4}(i \cos (\pi / 5)) U_{4}(i \cos (2 \pi / 5)) \\
& =\prod_{h=1}^{2} \prod_{k=1}^{2}\left(4 \cos ^{2}(h \pi / 5)+4 \cos ^{2}(k \pi / 5)\right)
\end{aligned}
$$

where $U_{4}(x)=16 x^{4}-12 x^{2}+1$ is the fourth Chebyshev polynomial of the second kind.

The double-product in equation (1) is an instance of the famous formula due to Kasteleyn [20] and to Temperley and Fisher [45] for the number of domino tilings of a $2 m \times 2 n$ checkerboard:

$$
\prod_{h=1}^{m} \prod_{k=1}^{n}\left(4 \cos ^{2} \frac{h \pi}{2 m+1}+4 \cos ^{2} \frac{k \pi}{2 n+1}\right),
$$

for which Theorem 15 provides a new proof.

In the case of the even $\times$ odd grid, there is an extra "twist": the double-product in Theorem 18 for the even $\times$ odd grid is (a slight re-writing of) the formula of Lu and $\mathrm{Wu}[25]$ for the number of domino tilings of a checkerboard on a Möbius strip.

Main idea. To sketch the main idea behind the proofs of the tiling theorems, suppose a group $G$ acts on a graph $\Gamma$ with fixed sink vertex (cf. Section 2.2). To study symmetric configurations with respect to the action of $G$, one considers a new firing rule in which a vertex only fires simultaneously with all other vertices in its orbit under $G$. This new firing rule can be encoded in an $m \times m$ matrix $D$ where $m$ is the number of orbits of nonsink vertices of $G$. We show in Corollary 11 that $\operatorname{det}(D)$ is the number of symmetric recurrents on $G$. Suppose, as is the case for sandpile grid graphs, that either $D$ or its transpose happens to be the (reduced) Laplacian of an associated graph $\Gamma^{\prime}$. Then the graph $\Gamma^{\prime}$ may be thought of as a quotient graph in the sense that its nonsink vertices correspond to the orbits of vertices of the original graph. The well-known matrix-tree theorem says that the determinant of $D$ is the number of spanning trees of $\Gamma^{\prime}$. Then the generalized Temperley bijection [21] says these spanning trees correspond with perfect matchings of a third graph $\Gamma^{\prime \prime}$. In this way, the symmetric recurrents on $\Gamma$ can be put into correspondence with the perfect matchings of $\Gamma^{\prime \prime}$. In the case where $\Gamma$ is a sandpile grid graph, $\Gamma^{\prime \prime}$ is a weighted grid graph, and perfect matchings of it correspond to weighted tilings of a checkerboard. Also, in this case, the matrix $D$ has a nice block triangular form (cf. Lemma 14), which leads to a recursive formula for its determinant and a connection with Chebyshev polynomials.

\section{Sandpiles}

\section{$2.1 \quad$ Basics}

In this section, we recall the basic theory of sandpile groups. The sandpile grid graph has multiple edges connecting its corner vertices to the sink vertex. Further, the creation of 
the quotient graphs required to count symmetric recurrent configurations on grid graphs in general gives rise to directed graphs (cf. Figure 15 in the proof of Theorem 18, for example). Therefore, the sandpile model we use here is for directed multigraphs. The reader is referred to [16] for a detailed exposition. Let $\Gamma=(V, E$, wt, $s)$ be a directed graph with vertices $V$, edges $E$, edge-weight function wt: $V \times V \rightarrow \mathbb{N}:=\{0,1,2, \ldots\}$, and special vertex $s \in V$. For each pair $v, w \in V$, we think of $\operatorname{wt}(v, w)$ as the number of edges running from $v$ to $w$. In particular, $\operatorname{wt}(v, w)>0$ if and only if $(v, w) \in E$. The vertex $s$ is called the sink of $\Gamma$, and it is assumed that each vertex of $\Gamma$ has a directed path to $s$. Let $\widetilde{V}:=V \backslash\{s\}$ be the set of non-sink vertices. A (sandpile) configuration on $\Gamma$ is an element of $\mathbb{N} \widetilde{V}$, the free commutative monoid on $\widetilde{V}$. If $c=\sum_{v \in \widetilde{V}} c_{v} v$ is a configuration, we think of each component, $c_{v}$, as a number of grains of sand stacked on vertex $v$. The vertex $v \in \widetilde{V}$ is unstable in $c$ if $c_{v} \geqslant \operatorname{outdeg}(v)$ where $\operatorname{outdeg}(v):=\sum_{w \in V} \operatorname{wt}(v, w)$, is the out-degree of $v$, i.e., the number of directed edges emanating from $v$. If $v$ is unstable in $c$, we may fire (topple) $c$ at $v$ to get a new configuration $c^{\prime}$ defined for each $w \in \widetilde{V}$ by

$$
c_{w}^{\prime}= \begin{cases}c_{v}-\operatorname{outdeg}(v)+\operatorname{wt}(v, v) & \text { if } w=v \\ c_{w}+\operatorname{wt}(v, w) & \text { if } w \neq v .\end{cases}
$$

In other words,

$$
c^{\prime}=c-\operatorname{outdeg}(v) v+\sum_{w \in \tilde{V}} \mathrm{wt}(v, w) w .
$$

If the configuration $\tilde{c}$ is obtained from $c$ by a sequence of firings of unstable vertices, we write

$$
c \rightarrow \tilde{c} .
$$

Since each vertex has a path to the sink, $s$, it turns out that by repeatedly firing unstable vertices each configuration relaxes to a stable configuration. Moreover, this stable configuration is independent of the ordering of firing of unstable vertices. Thus, we may talk about the stabilization of a configuration $c$, which we denote by $c^{\circ}$. Define the binary operation of stable addition on the set of all configurations as component-wise addition followed by stabilization. In other words, the stable addition of configurations $a$ and $b$ is given by

$$
(a+b)^{\circ}
$$

Let $\mathcal{M}$ denote the collection of stable configurations on $\Gamma$. Then stable addition restricted to $\mathcal{M}$ makes $\mathcal{M}$ into a commutative monoid.

A configuration $c$ on $\Gamma$ is recurrent if: (1) it is stable, and (2) given any configuration $a$, there is a configuration $b$ such that $(a+b)^{\circ}=c$. The maximal stable configuration, $c_{\max }$, is defined by

$$
c_{\max }:=\sum_{v \in \widetilde{V}}(\operatorname{outdeg}(v)-1) v .
$$

It turns out that the collection of recurrent configurations forms a principal semi-ideal of $\mathcal{M}$ generated by $c_{\max }$. This means that the recurrent configurations are exactly those obtained by adding sand to the maximal stable configuration and stabilizing. Further, 
the collection of recurrent configurations forms a group, $\mathcal{S}(\Gamma)$, called the sandpile group for $\Gamma$. Note that the identity for $\mathcal{S}(\Gamma)$ is not usually the zero-configuration, $\overrightarrow{0} \in \mathbb{N} \widetilde{V}$.

For an undirected graph, i.e., a graph for which $\operatorname{wt}(u, v)=\operatorname{wt}(v, u)$ for each pair of vertices $u$ and $v$, one may use the burning algorithm, due to Dhar [10], to determine whether a configuration is recurrent (for a generalization to directed graphs, see [43]):

Theorem 1 ([10],[16, Lemma 4.1]). Let c be a stable configuration on an undirected graph $\Gamma$. Define the burning configuration on $\Gamma$ to be the configuration obtained by firing the sink vertex:

$$
b:=\sum_{v \in \widetilde{V}} \mathrm{wt}(s, v) v .
$$

Then in the stabilization of $b+c$, each vertex fires at most once, and the following are equivalent:

1. $c$ is recurrent;

2. $(b+c)^{\circ}=c$;

3. in the stabilization of $b+c$, each non-sink vertex fires.

Define the proper Laplacian, $L: \mathbb{Z}^{V} \rightarrow \mathbb{Z}^{V}$, of $\Gamma$ by

$$
L(f)(v):=\sum_{w \in V} \operatorname{wt}(v, w)(f(v)-f(w))
$$

for each function $f \in \mathbb{Z}^{V}$. Taking the $\mathbb{Z}$-dual (applying the functor $\operatorname{Hom}(\cdot, \mathbb{Z})$ ) gives the mapping of free abelian groups

$$
\Delta: \mathbb{Z} V \rightarrow \mathbb{Z} V
$$

defined on vertices $v \in V$ by

$$
\Delta(v)=\operatorname{outdeg}(v) v-\sum_{w \in V} \operatorname{wt}(v, w) w .
$$

We call $\Delta$ the Laplacian of $\Gamma$. Restricting $\Delta$ to $\mathbb{Z} \widetilde{V}$ and setting the component of $s$ equal to 0 gives the reduced Laplacian, $\widetilde{\Delta}: \mathbb{Z} \widetilde{V} \rightarrow \mathbb{Z} \widetilde{V}$. If $v$ is an unstable vertex in a configuration $c$, firing $v$ gives the new configuration

$$
c-\widetilde{\Delta} v
$$

There is a well-known isomorphism

$$
\begin{aligned}
\mathcal{S}(\Gamma) & \rightarrow \mathbb{Z} \widetilde{V} / \operatorname{image}(\widetilde{\Delta}) \\
c & \mapsto c .
\end{aligned}
$$

While there may be many stable configurations in each equivalence class of $\mathbb{Z} \widetilde{V}$ modulo image $(\widetilde{\Delta})$, there is only one that is recurrent. For instance, the recurrent element in the equivalence class of $\overrightarrow{0}$ is the identity of $\mathcal{S}(\Gamma)$. 
A spanning tree of $\Gamma$ rooted at $s$ is a directed subgraph containing all the vertices, having no directed cycles, and for which $s$ has no out-going edges while every other vertex has exactly one out-going edge. The weights of the edges of a spanning tree are the same as they are for $\Gamma$, and the weight of a spanning tree is the product of the weights of its edges. The matrix-tree theorem says the sum of the weights of the set of all spanning trees of $\Gamma$ rooted at $s$ is equal to $\operatorname{det} \widetilde{\Delta}$, the determinant of the reduced Laplacian. It then follows from (2) that the number of elements of the sandpile group is also the sum of the weights of the spanning trees rooted at $s$.

\subsection{Symmetric configurations}

Preliminary versions of the results in this section appear in [12]. Let $G$ be a finite group. An action of $G$ on $\Gamma$ is an action of $G$ on $V$ fixing $s$, sending edges to edges, and preserving edge-weights. In detail, it is a mapping

$$
\begin{aligned}
G \times V & \rightarrow V \\
(g, v) & \mapsto g v
\end{aligned}
$$

satisfying

1. if $e$ is the identity of $G$, then $e v=v$ for all $v \in V$;

2. $g(h v)=(g h) v$ for all $g, h \in G$ and $v \in V$;

3. $g s=s$ for all $g \in G$;

4. if $(v, w) \in E$, then $(g v, g w) \in E$ and both edges have the same weight.

Note that these conditions imply that $\operatorname{outdeg}(v)=\operatorname{outdeg}(g v)$ for all $v \in V$ and $g \in G$. For the rest of this section, let $G$ be a group acting on $\Gamma$.

By linearity, the action of $G$ extends to an action on $\mathbb{N} V$ and $\mathbb{Z} V$. Since $G$ fixes the sink, $G$ acts on configurations and each element of $G$ induces an automorphism of $\mathcal{S}(\Gamma)$ (cf. 3). We say a configuration $c$ is symmetric (with respect to the action by $G$ ) if $g c=c$ for all $g \in G$.

Proposition 2. The action of $G$ commutes with stabilization. That is, if $c$ is any configuration on $\Gamma$ and $g \in G$, then $g\left(c^{\circ}\right)=(g c)^{\circ}$.

Proof. Suppose that $c$ is stabilized by firing the sequence of vertices $v_{1}, \ldots, v_{t}$. Then

$$
c^{\circ}=c-\sum_{i=1}^{t} \widetilde{\Delta} v_{i} .
$$

At the $k$-th step in the stabilization process, $c$ has relaxed to the configuration $c^{\prime}:=$ $c-\sum_{i=1}^{k} \widetilde{\Delta} v_{i}$. A vertex $v$ is unstable in $c^{\prime}$ if and only if $g v$ is unstable in $g c^{\prime}=g c-$ 
$\sum_{i=1}^{k} \widetilde{\Delta}\left(g v_{i}\right)$. Thus, we can fire the sequence of vertices $g v_{1}, \ldots, g v_{t}$ in $g c$, resulting in the stable configuration

$$
(g c)^{\circ}=g c-\sum_{i=1}^{t} \widetilde{\Delta}\left(g v_{i}\right) .
$$

Corollary 3. The action of $G$ preserves recurrent configurations, i.e., if c is a recurrent configuration and $g \in G$, then gc is recurrent.

Proof. If $c$ is recurrent, we can find a configuration $b$ such that $c=\left(b+c_{\max }\right)^{\circ}$. Then,

$$
g c=g\left(b+c_{\max }\right)^{\circ}=\left(g b+g c_{\max }\right)^{\circ}=\left(g b+c_{\max }\right)^{\circ} .
$$

Hence, $g c$ is recurrent.

Corollary 4. If $c$ is a symmetric configuration, then so is its stabilization.

Proof. For all $g \in G$, if $g c=c$, then $g\left(c^{\circ}\right)=(g c)^{\circ}=c^{\circ}$.

Remark 5. In fact, if $c$ is a symmetric configuration, one may find a sequence of symmetric configurations, $c_{1}, \ldots, c_{t}$ with $c_{t}=c^{\circ}$ such that $c \rightarrow c_{1} \rightarrow \cdots \rightarrow c_{t}$. This follows since in a symmetric configuration a vertex $v$ is unstable if and only if $g v$ is unstable for all $g \in G$. To construct $c_{i+1}$ from $c_{i}$, simultaneously fire all unstable vertices of $c_{i}$ (an alternative is to pick any vertex $v$, unstable in $c_{i}$, and simultaneously fire the vertices in $\{g v: g \in G\}$ ).

Proposition 6. The collection of symmetric recurrent configurations forms a subgroup of the sandpile group $\mathcal{S}(\Gamma)$.

Proof. Since the group action respects addition in $\mathbb{N} \widetilde{V}$ and stabilization, the sum of two symmetric recurrent configurations is again symmetric and recurrent. There is at least one symmetric recurrent configuration, namely, $c_{\max }$. Since the sandpile group is finite, it follows that these configurations form a subgroup.

Notation 7 . The subgroup of symmetric recurrent configurations on $\Gamma$ with respect to the action of the group $G$ is denoted $\mathcal{S}(\Gamma)^{G}$.

Proposition 8. If $c$ is symmetric and recurrent then $c=\left(a+c_{\max }\right)^{\circ}$ for some symmetric configuration a.

Proof. By [43] there exists an element $b$ in the image of $\widetilde{\Delta}$ such that: (1) $b_{v} \geqslant 0$ for all $v \in \widetilde{V}$, and (2) for each vertex $w \in \widetilde{V}$, there is a directed path to $w$ from some $v \in \widetilde{V}$ such that $b_{v}>0$, i.e., from some $v$ in the support of $b$. (If $\Gamma$ is undirected, one may find such a $b$ by applying $\widetilde{\Delta}$ to the vector whose components are all $1 \mathrm{~s})$. Define

$$
b^{G}=\sum_{g \in G} g b .
$$


Then $b^{G}$ is symmetric and equal to zero modulo the image of $\widetilde{\Delta}$. Take a large positive integer $N$ and consider $N b^{G}$, the vertex-wise addition of $b^{G}$ with itself $N$ times without stabilizing. Every vertex of $\Gamma$ is connected by a path from a vertex in the support of $b$, and hence, the same is true of $N b^{G}$. Thus, by choosing $N$ large enough and by firing symmetric vertices of $N b^{G}$, we obtain a symmetric configuration $b^{\prime}$ such that $b_{v}^{\prime} \geqslant c_{\max , v}$ for all $v$ and such that $b^{\prime}$ is zero modulo the image of $\widetilde{\Delta}$. Define $a=b^{\prime}-c_{\max }+c$, by construction a symmetric configuration. The unique recurrent element in the equivalence class of $b^{\prime}+c$ modulo the image of $\widetilde{\Delta}$ is $c$. Therefore,

$$
\left(a+c_{\max }\right)^{\circ}=\left(b^{\prime}+c\right)^{\circ}=c .
$$

The orbit of $v \in V$ under $G$ is the set

$$
G v=\{g v: g \in G\} .
$$

Let $\mathcal{O}=\mathcal{O}(\Gamma, G)=\{G v: v \in \widetilde{V}\}$ denote the set of orbits of the non-sink vertices. The symmetrized reduced Laplacian is the $\mathbb{Z}$-linear mapping

$$
\widetilde{\Delta}^{G}: \mathbb{Z O} \rightarrow \mathbb{Z} \mathcal{O}
$$

such that for all $v, w \in \widetilde{V}$, the $G w$-th component of $\widetilde{\Delta}^{G}(G v)$ is

$$
\left(\sum_{u \in G v} \widetilde{\Delta}(u)\right)_{w} .
$$

Remark 9. If $c \in \mathbb{Z} \widetilde{V}$ is symmetric, then define $[c] \in \mathbb{Z} \mathcal{O}$ by $[c]_{G v}:=c_{v}$ for all $v \in \widetilde{V}$, thus obtaining a bijection between symmetric elements of $\mathbb{Z} \widetilde{V}$ and $\mathbb{Z} \mathcal{O}$. The mapping $\widetilde{\Delta}^{G}$ is defined so that if $c$ is a symmetric configuration and $v \in \widetilde{V}$, then $[c]-\widetilde{\Delta}^{G}(G v)$ is the element of $\mathbb{Z} \mathcal{O}$ corresponding to

$$
c-\widetilde{\Delta}\left(\sum_{w \in G v} w\right)
$$

the symmetric configuration obtained from $c$ by firing all vertices in the orbit of $v$.

For the following let $r: \mathbb{Z} \widetilde{V}$ image $(\widetilde{\Delta}) \rightarrow \mathcal{S}(\Gamma)$ denote the inverse of the isomorphism in $(2)$.

Proposition 10. There is an isomorphism of groups,

$$
\phi: \mathbb{Z} \mathcal{O} / \operatorname{image}\left(\widetilde{\Delta}^{G}\right) \rightarrow \mathcal{S}(\Gamma)^{G},
$$

determined by $G v \mapsto r\left(\sum_{w \in G v} w\right)$ for $v \in \widetilde{V}$. 
Proof. The homomorphism $\lambda: \mathbb{Z O} \rightarrow \mathbb{Z} \widetilde{V}$ determined by

$$
\lambda(G v):=\sum_{w \in G v} w
$$

for $v \in \widetilde{V}$ induces the (well-defined) mapping

$$
\Lambda: \mathbb{Z O} / \operatorname{image}\left(\widetilde{\Delta}^{G}\right) \rightarrow \mathbb{Z} \widetilde{V} / \text { image }(\widetilde{\Delta}) .
$$

To see that the image of $r \circ \Lambda$ is symmetric, consider the symmetric configuration $|\mathcal{S}(\Gamma)|$. $c_{\max } \in \mathbb{Z} \widetilde{V}$, a configuration in the image of $\widetilde{\Delta}$. For each $v \in \widetilde{V}$,

$$
\phi(G v)=r(\Lambda(G v))=\left(|\mathcal{S}(\Gamma)| \cdot c_{\max }+\lambda(G v)\right)^{\circ},
$$

which is symmetric by Corollary 4 .

The mapping $c \mapsto[c]$, introduced in Remark 9, is a left inverse to $\lambda$. Thus, if $c \in \mathcal{S}(\Gamma)^{G}$, then $\phi([c])=c$, and hence $\phi$ is surjective. To show that $\phi$ is injective, it suffices to show that $\Lambda$ is injective. So suppose that $a=\lambda(o)$ for some $o \in \mathbb{Z} \mathcal{O}$ and that $a=\widetilde{\Delta}(b)$ for some $b \in \mathbb{Z} \widetilde{V}$. Fix $g \in G$, and consider the isomorphism $g: \mathbb{Z} \widetilde{V} \rightarrow \mathbb{Z} \widetilde{V}$ determined by the action of $g$ on vertices. A straightforward calculation shows that $\widetilde{\Delta}=g \widetilde{\Delta} g^{-1}$. It follows that

$$
\widetilde{\Delta}(b)=a=g a=g \widetilde{\Delta} b=\left(g \widetilde{\Delta} g^{-1}\right)(g b)=\widetilde{\Delta}(g b) .
$$

Since $\widetilde{\Delta}$ is invertible, it follows that $b=g b$ for all $g \in G$, i.e., $b$ is symmetric. Hence, $o=[a]=\widetilde{\Delta}^{G}([b])$, as required.

Corollary 11. The number of symmetric recurrent configurations is

$$
\left|\mathcal{S}(\Gamma)^{G}\right|=\operatorname{det} \widetilde{\Delta}^{G} .
$$

Remark 12. We have not assumed that the action of $G$ on $\Gamma$ is faithful. If $K$ is the kernel of the action of $G$, then $\mathcal{O}(\Gamma, G)=\mathcal{O}(\Gamma, G / K)$ and $\mathcal{S}^{G}=\mathcal{S}^{G / K}$. We also have $\widetilde{\Delta}^{G}=\widetilde{\Delta}^{G / K}$.

Example 13. Consider the graph $\Gamma$ of Figure 2 with sink $s$ and with each edge having weight 1.



Figure 2: The graph $\Gamma$ for Example 13.

Let $G=\{e, g\}$ be the group of order 2 with identity $e$. Consider the action of $G$ on $\Gamma$ for which $g$ swaps vertices $u$ and $v$ and fixes vertices $w$ and $s$. Ordering the vertices of $\Gamma$ as $u, v, w$ and ordering the orbits, $\mathcal{O}$, as $G u, G w$, the reduced Laplacian and the symmetrized reduced Laplacian for $\Gamma$ become 


$$
\widetilde{\Delta}=\left[\begin{array}{rrr}
3 & -1 & -1 \\
-1 & 3 & -1 \\
-1 & -1 & 2
\end{array}\right], \quad \widetilde{\Delta}^{G}=\left[\begin{array}{rr}
2 & -1 \\
-2 & 2
\end{array}\right]
$$

where we have labeled the columns by their corresponding vertices or orbits for convenience. To illustrate how one would compute the columns of the symmetrized reduced Laplacian in general, consider the column of $\widetilde{\Delta}^{G}$ corresponding to $G u=\{u, v\}$. It was computed by first adding the $u$ - and $v$-columns of $\widetilde{\Delta}$ to get the 3 -vector $\ell=(2,2,-2)$, then taking the $u$ and $w$ components of $\ell$ since $u$ and $w$ were chosen as orbit representatives.

There are $8=\operatorname{det} \widetilde{\Delta}$ recurrent elements $\left(c_{u}, c_{v}, c_{w}\right)$ of $\Gamma$ :

$$
(0,2,1),(1,2,0),(1,2,1),(2,0,1),(2,1,0),(2,1,1),(2,2,0),(2,2,1),
$$

and $(2,2,0)$ is the identity of $\mathcal{S}(\Gamma)$. In accordance with Corollary 11 , there are $2=\operatorname{det} \widetilde{\Delta}^{G}$ symmetric recurrent elements: $(2,2,0)$ and $(2,2,1)$.

\section{Matchings and trees}

In this section, assume that $\Gamma=(V, E$, wt, $s)$ is embedded in the plane, and fix a face $f_{s}$ containing the sink vertex, $s$. In $\S 4$ and $\S 5$, we always take $f_{s}$ to be the unbounded face. We recall the generalized Temperley bijection, due to [21], between directed spanning trees of $\Gamma$ rooted at $s$ and perfect matchings of a related weighted undirected graph, $\mathcal{H}(\Gamma)$. (The graph $\mathcal{H}(\Gamma)$ would be denoted $\mathcal{H}\left(s, f_{s}\right)$ in [21].)

It is sometimes convenient to allow an edge $e=(u, v)$ to be represented in the embedding by distinct weighted edges $e_{1}, \ldots, e_{k}$, each with tail $u$ and head $v$, such that $\sum_{i=1}^{k} \operatorname{wt}\left(e_{i}\right)=\operatorname{wt}(e)$. Also, we would like to be able to embed a pair of oppositely oriented edges between the same vertices so that they coincide in the plane. For these purposes then, we work in the more general category of weighted directed multi-graphs by allowing $E$ to be a multiset of edges in which an edge $e$ with endpoints $u$ and $v$ is represented as the set $e=\{u, v\}$ with a pair of weights $\operatorname{wt}(e,(u, v))$ and $\operatorname{wt}(e,(v, u))$, at least one of which is nonzero. Each edge in the embedding is then represented by a double-headed arrow with two weight labels (the label wt $(e,(u, v))$ being placed next to the head vertex, $v)$. Figure 3 shows a pair of edges $e=\{u, v\}$ and $e^{\prime}=\{u, v\}$ where $\operatorname{wt}(e,(u, v))=2$, $\operatorname{wt}(e,(v, u))=0, \operatorname{wt}\left(e^{\prime},(u, v)\right)=3$, and $\operatorname{wt}\left(e^{\prime},(v, u)\right)=1$. The top edge, $e$, represents a single directed edge $(u, v)$ of weight 2 , and the bottom edge represents a pair of directed edges of weights 3 and 1 . The two edges combine to represent a pair of directed edges, $(u, v)$ of weight 5 and $(v, u)$ of weight 1 .

The rough idea of the construction of the weighted undirected graph $\mathcal{H}(\Gamma)$ is to overlay the embedded graph $\Gamma$ with its dual, forgetting the orientation of the edges and introducing new vertices where their edges cross. Then remove $s$ and the vertex corresponding to the chosen face $f_{s}$, and remove their incident edges. In detail, the vertices of $\mathcal{H}(\Gamma)$ are

$$
V_{\mathcal{H}(\Gamma)}:=\left\{t_{v}: v \in V \backslash\{s\}\right\} \cup\left\{t_{e}: e \in E\right\} \cup\left\{t_{f}: f \in F \backslash\left\{f_{s}\right\}\right\},
$$






Figure 3: Edges for a planar embedding of a weighted directed graph.

where $F$ is the set of faces of $\Gamma$, including the unbounded face, and the edges of $\mathcal{H}(\Gamma)$ are

$$
E_{\mathcal{H}(\Gamma)}:=\left\{\left\{t_{u}, t_{e}\right\}: u \in V \backslash\{s\}, u \in e \in E\right\} \cup\left\{\left\{t_{e}, t_{f}\right\}: e \in E, e \in f \in F \backslash\left\{f_{s}\right\}\right\} .
$$

The weight of each edge of the form $\left\{t_{u}, t_{e}\right\}$ with $e=\{u, v\} \in E$ is defined to be $\operatorname{wt}(e,(u, v))$, and the weight of each edge of the form $\left\{t_{e}, t_{f}\right\}$ with $f \in F$ is defined to be 1 .

Figure 4 depicts a graph $\Gamma$ embedded in the plane (for which the multiset $E$ is actually just a set). The graph displayed in the middle is the superposition of $\Gamma$ with its dual, $\Gamma^{\perp}$. The unbounded face is chosen as $f_{s}$. For convenience, its corresponding vertex is omitted from the middle graph, and its incident edges are only partially drawn.


Figure 4: Construction of $\mathcal{H}(\Gamma)$. (Unlabeled edges have weight 1.)

A perfect matching of a weighted undirected graph is a subset of its edges such that each vertex of the graph is incident with exactly one edge in the subset. The weight of a perfect matching is the product of the weights of its edges.

We now describe the weight-preserving bijection between perfect matchings of $\mathcal{H}(\Gamma)$ and directed spanning trees of $\Gamma$ rooted at $s$ due to [21]. Let $T$ be a directed spanning tree of $\Gamma$ rooted at $s$, and let $\widetilde{T}$ be the corresponding directed spanning tree of $\Gamma^{\perp}$, the dual of $\Gamma$, rooted at $f_{s}$. (The tree $\widetilde{T}$ is obtained by properly orienting the edges of $\Gamma^{\perp}$ that do not cross edges of $T$ in $\Gamma \cup \Gamma^{\perp}$.) The perfect matching of $\mathcal{H}(\Gamma)$ corresponding to $T$ consists of the following:

1. an edge $\left\{t_{u}, t_{e}\right\}$ of weight $\operatorname{wt}(e)$ for each $e=(u, v) \in T$; 
2. an edge $\left\{t_{f}, t_{e}\right\}$ of weight 1 for each $\tilde{e}=\left(f, f^{\prime}\right) \in \widetilde{T}$, where $e$ is the edge in $\Gamma$ crossed by $\tilde{e}$.

See Figure 5 for an example continuing the example from Figure 4.


Figure 5: A spanning tree of $\Gamma$ determines a dual spanning tree for $\Gamma^{\perp}$ and a perfect matching for $\mathcal{H}(\Gamma)$. (See Figure 4. Unlabeled edges have weight 1.)

As discussed in [21], although $\mathcal{H}(\Gamma)$ depends on the embedding of $\Gamma$ and on the choice of $f_{s}$, the number of spanning trees of $\Gamma$ rooted at $s$ (and hence, the number of perfect matchings of $\mathcal{H}(\Gamma)$ ), counted according to weight, does not change. In what follows, we will always choose $f_{s}$ to be the unbounded face.

\section{Symmetric recurrents on the sandpile grid graph}

The ordinary $m \times n$ grid graph is the undirected graph $\Gamma_{m \times n}$ with vertices $[m] \times[n]$ and edges $\left\{(i, j),\left(i^{\prime}, j^{\prime}\right)\right\}$ such that $\left|i-i^{\prime}\right|+\left|j-j^{\prime}\right|=1$. The $m \times n$ sandpile grid graph, $\mathrm{S} \Gamma_{m \times n}$, is formed from $\Gamma_{m \times n}$ by adding a (disjoint) sink vertex, $s$, then edges incident to $s$ so that every non-sink vertex of the resulting graph has degree 4 . For instance, each of the four corners of the sandpile grid graph shares an edge of weight 2 with $s$ in the case where $m \geqslant 2$ and $n \geqslant 2$, as on the left in Figure 6.

We embed $\Gamma_{m \times n}$ in the plane as the standard grid with vertices arranged as in a matrix, with $(1,1)$ in the upper left and $(m, n)$ in the lower right. We embed $\mathrm{S} \Gamma_{m \times n}$ similarly, but usually identify the sink vertex, $s$, with the unbounded face of $\Gamma_{m \times n}$ for convenience in drawing, as on the left-hand side in Figure 6. The edges leading to the sink are sometimes entirely omitted from the drawing, as in Figure 10.

In this section, symmetric recurrent will always refer to a recurrent element on $\mathrm{S}_{m \times n}$ with horizontal and vertical symmetry, i.e., an element of $\mathcal{S}\left(\mathrm{S}_{m \times n}\right)^{G}$ where $G$ is the Klein 4-group,

$$
G=\left\langle\sigma, \tau: \sigma^{2}=\tau^{2}=1, \sigma \tau=\tau \sigma\right\rangle
$$






$\mathrm{S} \Gamma_{4 \times 5}$

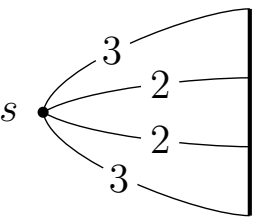

$\mathrm{S} \Gamma_{4 \times 1}$

Figure 6: Two sandpile grid graphs. (The sink for $\mathrm{S}_{4 \times 5}$ is not drawn.)

acting on $\mathrm{S} \Gamma_{m \times n}$ by

$$
\sigma(i, j)=(i, n-j+1), \quad \tau(i, j)=(m-i+1, j), \quad \text { and } \sigma(s)=\tau(s)=s .
$$

Our main goal in this section is to study the symmetric recurrent configurations on the sandpile grid graph. After collecting some basic facts about certain tridiagonal matrices, we divide the study into three cases: even $\times$ even-, even $\times$ odd-, and odd $\times$ odd-dimensional grids. In each case we provide a formula for the number of symmetric recurrents using Chebyshev polynomials and show how these configurations are related to domino tilings of various types of checkerboards.

\subsection{Some tridiagonal matrices.}

Recall that Chebyshev polynomials of the first kind are defined by the recurrence

$$
\begin{aligned}
& T_{0}(x)=1 \\
& T_{1}(x)=x \\
& T_{j}(x)=2 x T_{j-1}(x)-T_{j-2}(x) \quad \text { for } j \geqslant 2,
\end{aligned}
$$

and Chebyshev polynomials of the second kind are defined by

$$
\begin{aligned}
& U_{0}(x)=1 \\
& U_{1}(x)=2 x \\
& U_{j}(x)=2 x U_{j-1}(x)-U_{j-2}(x) \quad \text { for } j \geqslant 2 .
\end{aligned}
$$

Two references are [29] and [46].

It follows from the recurrences that these polynomials may be expressed as determinants of $j \times j$ tridiagonal matrices:

$$
T_{j}(x)=\operatorname{det}\left[\begin{array}{cccccc}
x & 1 & & & & \\
1 & 2 x & 1 & & & \\
& 1 & 2 x & 1 & & \\
& & & \ddots & & \\
& & & 1 & 2 x & 1 \\
& & & & 1 & 2 x
\end{array}\right], \quad U_{j}(x)=\operatorname{det}\left[\begin{array}{ccccccc}
2 x & 1 & & & & \\
1 & 2 x & 1 & & & \\
& 1 & 2 x & 1 & & \\
& & & \ddots & & \\
& & & 1 & 2 x & 1 \\
& & & & 1 & 2 x
\end{array}\right],
$$


and, hence, $T_{j}(-x)=(-1)^{j} T_{j}(x)$ and $U_{j}(-x)=(-1)^{j} U_{j}(x)$.

We have the well-known factorizations:

$$
\begin{aligned}
& T_{j}(x)=2^{j-1} \prod_{k=1}^{j}\left(x-\cos \left(\frac{(2 k-1) \pi}{2 j}\right)\right) \\
& U_{j}(x)=2^{j} \prod_{k=1}^{j}\left(x-\cos \left(\frac{k \pi}{j+1}\right)\right) .
\end{aligned}
$$

We will also use the following well-known identities:

$$
\begin{aligned}
T_{2 j}(x) & =T_{j}\left(2 x^{2}-1\right)=(-1)^{j} T_{j}\left(1-2 x^{2}\right) \\
2 T_{j}(x) & =U_{j}(x)-U_{j-2}(x) .
\end{aligned}
$$

Corollary 11 will be used to count the symmetric recurrents on sandpile grid graphs. The form of the determinant that arises is treated by the following.

Lemma 14. Let $m$ and $n$ be positive integers. Let $A, B$, and $C$ be $n \times n$ matrices over the complex numbers, and let $I_{n}$ be the $n \times n$ identity matrix. Define the $m n \times m n$ tridiagonal block matrix

$$
D(m)=\left[\begin{array}{ccccc}
A & -I_{n} & & & \\
-I_{n} & A & -I_{n} & & \\
& & \ddots & & \\
& & -I_{n} & A & -I_{n} \\
& & & -C & B
\end{array}\right],
$$

where the super-and sub-diagonal blocks are all $-I_{n}$ except for the one displayed block consisting of $-C$ and all omitted entries in the matrix are zero. Take $D(1)=B$. Then

$$
\operatorname{det} D(m)=(-1)^{n} \operatorname{det}(T)
$$

where

$$
T=-B U_{m-1}\left(\frac{1}{2} A\right)+C U_{m-2}\left(\frac{1}{2} A\right)
$$

letting $U_{-1}(x):=0$.

Proof. The case $m=1$ is immediate. For $m>1$, Theorem 2 of [31] gives a formula for calculating the determinant of a general tridiagonal block matrix. In our case, it says

$$
\operatorname{det} D(m)=(-1)^{n} \operatorname{det} E_{\boldsymbol{t}},
$$

where $E_{t}$ is the top-left block of size $n \times n$ of the matrix

$$
E:=\left[\begin{array}{cc}
-B & C \\
I_{n} & 0
\end{array}\right]\left[\begin{array}{cc}
A & -I_{n} \\
I_{n} & 0
\end{array}\right]^{m-2}\left[\begin{array}{cc}
A & I_{n} \\
I_{n} & 0
\end{array}\right]
$$


Set $S_{0}=I_{n}$, and for all positive integers $j$, define

$$
S_{j}=\left(\left[\begin{array}{cc}
A & -I_{n} \\
I_{n} & 0
\end{array}\right]^{j-1}\left[\begin{array}{cc}
A & I_{n} \\
I_{n} & 0
\end{array}\right]\right)_{t}
$$

and

$$
S_{j}^{\prime}=\left(\left[\begin{array}{cc}
A & -I_{n} \\
I_{n} & 0
\end{array}\right]^{j-1}\left[\begin{array}{cc}
A & I_{n} \\
I_{n} & 0
\end{array}\right]\right)_{b},
$$

where the subscripts $\boldsymbol{t}$ and $\boldsymbol{b}$ denote taking the top-left and bottom-left blocks of size $n \times n$, respectively. It follows that

$$
S_{0}=I_{n}, \quad S_{1}=A, \quad \text { and } \quad S_{j}=A S_{j-1}-S_{j-2} \text { for } j \geqslant 2,
$$

and

$$
S_{j}^{\prime}=S_{j-1} \text { for all } j \geqslant 1 .
$$

By (5) and (11), $S_{j}=U_{j}\left(\frac{1}{2} A\right)$. Hence,

$$
E_{t}=-B S_{m-1}+C S_{m-1}^{\prime}=-B U_{m-1}\left(\frac{1}{2} A\right)+C U_{m-2}\left(\frac{1}{2} A\right)
$$

as required.

\subsection{Symmetric recurrents on a $2 m \times 2 n$ sandpile grid graph.}

A checkerboard is a rectangular array of squares. A domino is a $1 \times 2$ or $2 \times 1$ array of squares and, thus, covers exactly two adjacent squares of the checkerboard. A domino tiling of the checkerboard consists of placing non-overlapping dominos on the checkerboard, covering every square. As is usually done, and exhibited in Figure 7, we identify domino tilings of an $m \times n$ checkerboard with perfect matchings of $\Gamma_{m \times n}$. Figure 8 exhibits the 36 domino tilings of a $4 \times 4$ checkerboard.



Figure 7: Correspondence between a perfect matching of $\Gamma_{3 \times 4}$ and a domino tiling of its corresponding checkerboard.

Part (4) of the following theorem is the well-known formula due to Kasteleyn [20] and to Temperley and Fisher [45] for the number of domino tilings of a checkerboard. We provide a new proof. 




Figure 8: The 36 domino tilings of a $4 \times 4$ checkerboard. The blue dominos are assigned weight 2 for the purposes of Theorem 18 .

Theorem 15. Let $U_{j}(x)$ denote the $j$-th Chebyshev polynomial of the second kind, and let

$$
\xi_{h, d}:=\cos \left(\frac{h \pi}{2 d+1}\right),
$$

for all integers $h$ and $d$. Then for all integers $m, n \geqslant 1$, the following are equal:

1. the number of symmetric recurrents on $\mathrm{S}_{2 m \times 2 n}$;

2. the number of domino tilings of a $2 m \times 2 n$ checkerboard;

3.

$$
(-1)^{m n} \prod_{h=1}^{m} U_{2 n}\left(i \xi_{h, m}\right) \text {; }
$$

4.

$$
\prod_{h=1}^{m} \prod_{k=1}^{n}\left(4 \xi_{h, m}^{2}+4 \xi_{k, n}^{2}\right) .
$$

Proof. It may be helpful to read Example 17 in parallel with this proof. 
Let $A_{n}=\left(a_{h, k}\right)$ be the $n \times n$ tridiagonal matrix with entries

$$
a_{h, k}=\left\{\begin{aligned}
4 & \text { if } h=k \neq n \\
3 & \text { if } h=k=n \\
-1 & \text { if }|h-k|=1 \\
0 & \text { if }|h-k| \geqslant 2
\end{aligned}\right.
$$

In particular, $A_{1}=[3]$. Take the vertices $[m] \times[n]$ as representatives for the orbits of $G$ acting the non-sink vertices of $\mathrm{S}_{2 m \times 2 n}$. Ordering these representatives lexicographically, i.e., left-to-right then top-to-bottom, the symmetrized reduced Laplacian (2.2) is given by the $m n \times m n$ tridiagonal block matrix

$$
\widetilde{\Delta}^{G}=\left[\begin{array}{ccccccc}
A_{n} & -I_{n} & & & \cdots & & 0 \\
-I_{n} & A_{n} & -I_{n} & & & & \\
& \ddots & \ddots & \ddots & & & \vdots \\
& & -I_{n} & A_{n} & -I_{n} & & \\
\vdots & & & \ddots & \ddots & \ddots & \\
& & & & -I_{n} & A_{n} & -I_{n} \\
0 & & \ldots & & & -I_{n} & B_{n}
\end{array}\right]
$$

where $I_{n}$ is the $n \times n$ identity matrix and $B_{n}:=A_{n}-I_{n}$. If $m=1$, then $\widetilde{\Delta}^{G}:=B_{n}$.

$[(\mathbf{1})=(2)]$ : The matrix $\widetilde{\Delta}^{G}$ is the reduced Laplacian of a sandpile graph we now describe. Let $D_{m \times n}$ be the graph obtained from $\Gamma_{m \times n}$, the ordinary grid graph, by adding (i) a sink vertex, $s^{\prime}$, (ii) an edge of weight 2 from the vertex $(1,1)$ to $s^{\prime}$, and (iii) edges of weight 1 from each of the other vertices along the left and top sides to $s^{\prime}$, i.e., $\left\{(h, 1), s^{\prime}\right\}$ for $1<h \leqslant m$ and $\left\{(1, k), s^{\prime}\right\}$ for $1<k \leqslant n$. We embed $D_{m \times n}$ in the plane so that the non-sink vertices form an ordinary grid, and the edge of weight 2 is represented by a pair of edges of weight 1, forming a digon. Then, $\mathcal{H}\left(D_{m \times n}\right)=\Gamma_{2 m \times 2 n}$ (see Figure 11).

Since $\widetilde{\Delta}^{G}=\widetilde{\Delta}_{D_{m \times n}}$, taking determinants shows that the number of symmetric recurrents on $\mathrm{S}_{2 m \times 2 n}$ is equal to the size of the sandpile group of $D_{m \times n}$, and hence to the number of spanning trees of $D_{m \times n}$ rooted at $s^{\prime}$, counted according to weight. These spanning trees are, in turn, in bijection with the perfect matchings of the graph $\mathcal{H}\left(D_{m \times n}\right)=\Gamma_{2 m \times 2 n}$ obtained from the generalized Temperley bijection of Section 3. Hence, the numbers in parts (1) and (2) are equal.

$[(\mathbf{1})=(\mathbf{3})]$ : By Corollary $11, \operatorname{det} \widetilde{\Delta}^{G}$ is the number of symmetric recurrents on $\mathrm{S}_{2 m \times 2 n}$. By Lemma 14,

$$
\operatorname{det} \widetilde{\Delta}^{G}=(-1)^{n} \operatorname{det}(T)
$$

where

$$
\begin{aligned}
T & =-B_{n} U_{m-1}\left(\frac{A_{n}}{2}\right)+U_{m-2}\left(\frac{A_{n}}{2}\right) \\
& =U_{m-1}\left(\frac{A_{n}}{2}\right)-U_{m}\left(\frac{A_{n}}{2}\right) .
\end{aligned}
$$


Using (7) and the fact that the Chebyshev polynomials of the second kind satisfy

$$
U_{j}(\cos \theta)=\frac{\sin ((j+1) \theta)}{\sin \theta},
$$

it is easy to check that the polynomial

$$
p(x):=U_{m}\left(\frac{x}{2}\right)-U_{m-1}\left(\frac{x}{2}\right)
$$

is a monic polynomial of degree $m$ with zeros

$$
t_{h, m}:=2 \cos \frac{(2 h+1) \pi}{2 m+1}, \quad 0 \leqslant h \leqslant m-1 .
$$

Thus,

$$
T=-p\left(A_{n}\right)=-\prod_{h=0}^{m-1}\left(A_{n}-t_{h, m} I_{n}\right)
$$

and by equation (13),

$$
\operatorname{det} \widetilde{\Delta}^{G}=\prod_{h=0}^{m-1} \chi_{n}\left(t_{h, m}\right),
$$

where $\chi_{n}(x)$ is the characteristic polynomial of $A_{n}$. Therefore, to show that the expressions in parts (1) and (3) are equal, it suffices to show that

$$
\chi_{n}\left(t_{h, m}\right)=(-1)^{n} U_{2 n}\left(i \xi_{m-h, m}\right)
$$

for each $h \in\{0,1, \cdots, m-1\}$, which one may do by showing that both sides of the equation satisfy the same recurrence.

$[(3)=(4)]$ : This equality follows from (7).

Example 16. Figure 9 lists the 36 symmetric recurrents on $\mathrm{S}_{4 \times 4}$ in no particular order. Given a symmetric recurrent, $c$, let $\tilde{c}$ be the restriction of $c$ to the vertices $(1,1),(1,2)$, $(2,1)$, and $(2,2)$, representing the orbits of the Klein 4-group action on $\mathrm{S}_{4 \times 4}$. We regard $\tilde{c}$ as a configuration on $D_{2 \times 2}$, the sandpile graph introduced in the proof of Theorem 15 . Let $\iota(c)$ be the recurrent element of the sandpile graph $D_{2 \times 2}$ equivalent to $\tilde{c}$ modulo the reduced Laplacian of $D_{2 \times 2}$. Then $c \mapsto \iota(c)$ determines a bijection between the symmetric recurrents of $\mathrm{S}_{4 \times 4}$ and the recurrents of $D_{2 \times 2}$. In [16], it is shown that the sandpile group of a graph acts freely and transitively on the set of spanning trees of the graph rooted at the sink, i.e., this set of spanning trees is a torsor for the sandpile group. Thus, via the Temperley bijection, the domino tilings of the $4 \times 4$ checkerboard, forms a torsor for the group of symmetric recurrents on $\mathrm{S}_{4 \times 4}$. 




Figure 9: The 36 symmetric recurrents on $\mathrm{S}_{4 \times 4}$.

Example 17. This example illustrates part of the proof of Theorem 15 for the case $m=4$ and $n=3$. Figure 10 shows the graph $\mathrm{S}_{8 \times 6}$. The boxed $4 \times 3$ block of vertices in the upper left are representatives of the orbits of the Klein 4-group action. Order these from left-to-right, top-to-bottom, to get the matrix for the symmetrized reduced Laplacian, $\widetilde{\Delta}_{8 \times 6}^{G}$. The vertex $(2,3)$ of $S \Gamma_{8}$ in Figure 10 is colored blue. If this vertex is fired simultaneously with the other vertices in its orbit, it will lose 4 grains of sand to its neighbors but gain 1 grain of sand from the adjacent vertex in its orbit. This firing-rule is encoded in the sixth column of $\widetilde{\Delta}_{8 \times 6}^{G}$ (shaded blue).



Figure 10: A sandpile grid graph and its symmetrized reduced Laplacian. 
The matrix $\widetilde{\Delta}_{8 \times 6}^{G}$ is the reduced Laplacian of the graph $D_{4 \times 3}$, shown in Figure 11. To form $\mathcal{H}\left(D_{4 \times 3}\right)=\Gamma_{8 \times 6}$, we first overlay $D_{4 \times 3}$ with its dual, as shown, then remove the vertices $s$ and $\tilde{s}$ and their incident edges. Figure 12 shows how a spanning tree of $D_{4 \times 3}$ (in black) determines a spanning tree of the dual graph (in blue) and a domino tiling of the $8 \times 6$ checkerboard.


Figure 11: The symmetrized reduced Laplacian for $\mathrm{S} \Gamma_{8 \times 6}$ is the reduced Laplacian for $D_{4 \times 3}$. Removing $s$ and $\tilde{s}$ and their incident edges from the graph on the right shows $\mathcal{H}\left(D_{4 \times 3}\right)=\Gamma_{8 \times 6}$.



Figure 12: Every domino tiling of an even-sided checkerboard consists of a spanning tree entwined with its dual spanning tree. 


\subsection{Symmetric recurrents on a $2 m \times(2 n-1)$ sandpile grid graph.}

The $m \times n$ Möbius grid graph, $\Gamma_{m \times n}^{\mathrm{mob}}$, is the graph formed from the ordinary $m \times n$ grid graph, $\Gamma_{m \times n}$, by adding the edges $\{(h, 1),(m-h+1, n)\}$ for $1 \leqslant h \leqslant m$. A Möbius checkerboard is an ordinary checkerboard with its left and right sides glued with a twist. Domino tilings of an $m \times n$ Möbius checkerboard are identified with perfect matchings of $\Gamma_{m \times n}^{\mathrm{mob}}$. See Figure 13 for examples.



(i)



(ii)

Figure 13: (i) The $4 \times 4$ Möbius grid graph, $\Gamma_{4 \times 4}^{\mathrm{mob}}$; (ii) A tiling of the $4 \times 4$ Möbius checkerboard.

As part of Theorem 18, we will show that the domino tilings of a $2 m \times 2 n$ Möbius checkerboard can be counted using weighted domino tilings of an associated ordinary checkerboard, which we now describe. Define the Möbius-weighted $m \times n$ grid graph, $\mathrm{M} \Gamma_{m \times n}$, as the ordinary $m \times n$ grid graph but with each edge of the form $\{(m-2 h, n-$ 1), $(m-2 h, n)\}$ for $0 \leqslant h<\left\lfloor\frac{m}{2}\right\rfloor$ assigned the weight 2 , and, if $m$ is odd, then in addition assign the edge $\{(1, n-1),(1, n)\}$ the weight 3 (and all other edges have weight 1 ). (In the case $m=1$, the weight of the edge $\{(1, n-1),(1, n)\}$ is be defined to be 3 .) See Figure 14 for examples. The Möbius-weighted $m \times n$ checkerboard is the ordinary $m \times n$

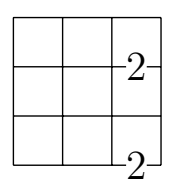

$\mathrm{M \Gamma}_{4 \times 4}$

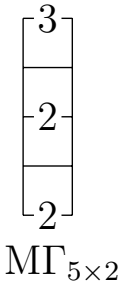

Figure 14: Two Möbius-weighted grid graphs.

checkerboard but for which the weight of a domino tiling is taken to be the weight of the corresponding perfect matching of $M \Gamma_{m \times n}$. In Figure 8, the dominos corresponding to edges of weight 2 are shaded. Thus, the first three tilings in the first row of Figure 8 have weights 4, 2, and 1, respectively. Example 22 considers a case for which $m$ is odd.

Theorem 18. Let $T_{j}(x)$ denote the $j$-th Chebyshev polynomial of the first kind, and let

$$
\xi_{h, d}:=\cos \left(\frac{h \pi}{2 d+1}\right) \quad \text { and } \quad \zeta_{h, d}:=\cos \left(\frac{(2 h-1) \pi}{4 d}\right)
$$


for all integers $h$ and $d \neq 0$. Then for all integers $m, n \geqslant 1$, the following are equal:

1. the number of symmetric recurrents on $\mathrm{S}_{2 m \times(2 n-1)}$;

2. if $n>1$, the number of domino tilings of the Möbius-weighted $2 m \times 2 n$ checkerboard, and if $n=1$, the number of domino tilings of the Möbius-weighted $(2 m-1) \times 2$ checkerboard, counted according to weight;

3.

$$
(-1)^{m n} 2^{m} \prod_{h=1}^{m} T_{2 n}\left(i \xi_{h, m}\right)
$$

4.

$$
\prod_{h=1}^{m} \prod_{k=1}^{n}\left(4 \xi_{h, m}^{2}+4 \zeta_{k, n}^{2}\right)
$$

5. the number of domino tilings of a $2 m \times 2 n$ Möbius checkerboard.

Remark 19. By identity (8),

$$
T_{2 n}\left(i \xi_{h, m}\right)=(-1)^{n} T_{n}\left(1+2 \xi_{h, m}^{2}\right),
$$

from which it follows, after proving Theorem 18, that

$$
2^{m} \prod_{h=1}^{m} T_{n}\left(1+2 \xi_{h, m}^{2}\right)
$$

is another way to express the numbers in parts (1)-(5).

Proof of Theorem 18. Except for two major exceptions - noted in the proof below and in Remark 20- the proof is similar to that of Theorem 15 after altering the definitions of the matrices $A_{n}$ and $B_{n}$ used there. This time, for $n>1$, let $A_{n}^{\prime}=\left(a_{h, k}^{\prime}\right)$ be the $n \times n$ tridiagonal matrix with entries

$$
a_{h, k}^{\prime}=\left\{\begin{aligned}
4 & \text { if } h=k \\
-1 & \text { if }|h-k|=1 \text { and } h \neq n \\
-2 & \text { if } h=n \text { and } k=n-1 \\
0 & \text { if }|h-k| \geqslant 2
\end{aligned}\right.
$$

In particular, $A_{1}^{\prime}=[4]$. Define the matrix $B_{n}^{\prime}=\left(b_{h, k}^{\prime}\right)$ by

$$
b_{h, k}^{\prime}= \begin{cases}3 & \text { if } h=k \\ a_{h, k}^{\prime} & \text { otherwise }\end{cases}
$$


Thus, for instance,

$$
A_{3}^{\prime}=\left[\begin{array}{rrr}
4 & -1 & 0 \\
-1 & 4 & -1 \\
0 & -2 & 4
\end{array}\right], \quad B_{3}^{\prime}=\left[\begin{array}{rrr}
3 & -1 & 0 \\
-1 & 3 & -1 \\
0 & -2 & 3
\end{array}\right]
$$

If $n=1$, take $A_{1}^{\prime}=[4]$ and $B_{1}^{\prime}=[3]$.

Reasoning as in the proof of Theorem 15, equation (12) with $A_{n}^{\prime}$ and $B_{n}^{\prime}$ substituted for $A_{n}$ and $B_{n}$ gives the symmetrized reduced Laplacian, $\widetilde{\Delta}^{G}$, of $\mathrm{S}_{2 m \times(2 n-1)}$. A major difference with the proof of Theorem 15 is that unless $n=1$, the matrix $\widetilde{\Delta}^{G}$ is not the reduced Laplacian matrix of a sandpile graph since the sum of the elements in its penultimate column is -1 whereas the sum of the elements in any column of the reduced Laplacian of a sandpile graph must be nonnegative. However, the transpose $\left(\widetilde{\Delta}^{G}\right)^{t}$ is the reduced Laplacian of a sandpile graph, which we call $D_{m \times n}^{\prime}$. We embed it in the plane as a grid as we did previously with $D_{m \times n}$ in the proof of Theorem 15, but this time with some edge-weights not equal to 1.

Figure 15 shows $D_{4 \times 3}^{\prime}$. It is the same as $D_{4 \times 3}$ as depicted in Figure 11, except that arrowed edges, $\longleftrightarrow$, have been substituted for certain edges. Each represents a pair of arrows - one from right-to-left of weight 2 and one from left-to-right of weight 1 embedded so that they coincide, as discussed in Section 3.



Figure 15: The symmetrized reduced Laplacian for $\mathrm{S}_{8 \times 5}$ is the reduced Laplacian for $D_{4 \times 3}^{\prime}$. Arrowed edges each represent a pair of directed edges of weights 1 and 2 , respectively, as indicated by the number of arrow heads. All other edges have weight 1 .

Reasoning as in the proof of Theorem 15, we see that the number of perfect matchings of $\mathcal{H}\left(D_{m \times n}^{\prime}\right)$ is equal to the number of perfect matchings of $\mathrm{M}_{2 m \times(2 n-1)}$, each counted according to weight. This number is $\operatorname{det}\left(\widetilde{\Delta}^{G}\right)^{t}=\operatorname{det} \widetilde{\Delta}^{G}$, which is the number of symmetric recurrents on $\mathrm{S}_{2 m \times(2 n-1)}$ by Corollary 11 .

The equality between the formulas in (4) and (5) is established using the $\mathrm{Lu}-\mathrm{Wu}$ 
formula ((2) in [25]) for the number of domino tilings of the $2 m \times 2 n$ Möbius checkerboard:

$$
\prod_{h=1}^{m} \prod_{k=1}^{n}\left(4 \xi_{h, m}^{2}+4 \mu_{k, n}^{2}\right),
$$

where $\mu_{k, n}:=\sin ((4 k-1) \pi /(4 n))$. This formula is identical to our double-product in part (4) up to a trigonometric identity.

Remark 20. In the proof of Theorem 18, we rewrote the double-product in part (4) as the Lu-Wu formula ((2) in [25]) for the number of domino tilings of the $2 m \times 2 n$ Möbius checkerboard. Thus, it is the work of $\mathrm{Lu}$ and $\mathrm{Wu}$ that allowed us to add part (5) to Theorem 18. This is in contrast to Theorem 15, which gave an independent proof of the Kastelyn and Temperley-Fisher formula for the number of tilings of the ordinary $2 m \times 2 n$ checkerboard.

Example 21. The 36 tilings of the ordinary $4 \times 4$ checkerboard are listed in Figure 8 . Considering these as tilings of the Möbius-weighted $4 \times 4$ checkerboard, the sum of the weights of the tilings is 71 , which is the number of tilings of the $4 \times 4$ Möbius checkerboard and the number of symmetric recurrents on $\mathrm{S}_{4 \times 3}$, in accordance with Theorem 18 .

Example 22. Figure 16 shows the domino tilings of the Möbius-weighted $5 \times 2$ checkerboard. The total number of tilings, counted according to weight, is 41 , which is the number of domino tilings of a $6 \times 2$ Möbius checkerboard, in agreement with case $m=3$ and $n=1$ of Theorem 18 .



12



6



6



3



6



4

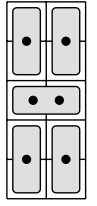

2

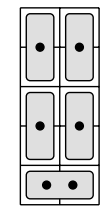

2

Figure 16: Domino tilings of the Möbius-weighted $5 \times 2$ checkerboard. The number of dots on each domino indicates its weight. The weight of each tiling appears underneath.

\subsection{Symmetric recurrents on a $(2 m-1) \times(2 n-1)$ sandpile grid graph.}

The 2-weighted $2 m \times 2 n$ grid graph, $2-\Gamma_{2 m \times 2 n}$ is the ordinary $2 m \times 2 n$ grid graph but where each horizontal edge of the form $\{(2 m-2 h, 2 n-1),(2 m-2 h, 2 n)\}$ for $0 \leqslant h<m$ and each vertical edge of the form $\{(2 m-1,2 n-2 k),(2 m, 2 n-2 k)\}$ for $0 \leqslant k<n$ is assigned the weight 2 (and all other edges have weight 1). See Figure 14 for an example. The 2 -weighted $2 m \times 2 n$ checkerboard is the ordinary $2 m \times 2 n$ checkerboard but for which the weight of a domino tiling is taken to be the weight of the corresponding perfect matching of $2-\Gamma_{2 m \times 2 n}$. 


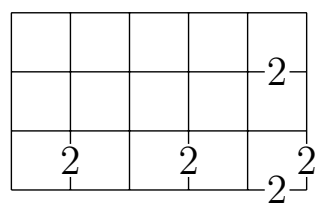

$2-\Gamma_{4 \times 6}$

Figure 17: A 2-weighted grid graph.

Theorem 23. Let $T_{j}(x)$ denote the $j$-th Chebyshev polynomial of the first kind, and let

$$
\zeta_{h, d}:=\cos \left(\frac{(2 h-1) \pi}{4 d}\right)
$$

for all integers $h$ and $d \neq 0$. Then for all integers $m, n \geqslant 1$, the following are equal:

1. the number of symmetric recurrents on $\mathrm{S} \Gamma_{(2 m-1) \times(2 n-1)}$;

2. the number of domino tilings of the 2-weighted checkerboard of size $2 m \times 2 n$;

3.

$$
(-1)^{m n} 2^{m} \prod_{h=1}^{m} T_{2 n}\left(i \zeta_{h, m}\right) \text {; }
$$

4.

$$
\prod_{h=1}^{m} \prod_{k=1}^{n}\left(4 \zeta_{h, m}^{2}+4 \zeta_{k, n}^{2}\right)
$$

Remark 24. As in Remark 19, we use identity (8), this time to get

$$
T_{2 n}\left(i \zeta_{h, m}\right)=(-1)^{n} T_{n}\left(1+2 \zeta_{h, m}^{2}\right),
$$

allowing us to equate the formula in part (3) with

$$
2^{m} \prod_{h=1}^{m} T_{n}\left(1+2 \zeta_{h, m}^{2}\right) .
$$

(We do not know of an analogous expression for the formula in Theorem 15 (3) in terms of products of $n$-th Chebyshev polynomials.)

Proof. The proof is similar to those for Theorem 15 and Theorem 18. Let $A_{n}^{\prime}$ be the matrix defined at the beginning of the proof of Theorem 18. Then the symmetrized reduced Laplacian, $\widetilde{\Delta}^{G}$, for $2-\Gamma_{(2 m-1) \times(2 n-1)}$ is the matrix $D(m)$ displayed in the statement of Lemma 14 after setting $A=B=A_{n}^{\prime}$ and $C=2 I_{n}$.

The transpose $\left(\widetilde{\Delta}^{G}\right)^{t}$ is the reduced Laplacian of a sandpile graph, which we denote by $D_{m \times n}^{\prime \prime}$ and embed in the plane as we did previously for $D_{m \times n}$ and $D_{m \times n}^{\prime}$ in Theorems 15 and 18. The embedding of $D_{m \times n}^{\prime \prime}$ differs from that of $D_{m \times n}^{\prime}$ only in that each edge of the form $((m, i),(m-1, i))$ where $i \in[n]$ now carries weight 2 , again embedded as one edge coincident with the edge $((m-1, i),(m, i))$ in the plane (Figure 18 displays $\left.D_{4 \times 3}^{\prime \prime}\right)$. 


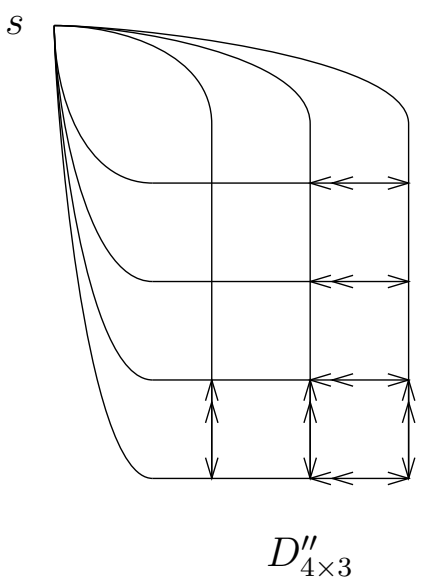

Figure 18: The symmetrized reduced Laplacian for $\mathrm{S}_{7 \times 5}$ is the reduced Laplacian for $D_{4 \times 3}^{\prime \prime}$. (The edge weights are encoded as in Figure 15).

\section{The order of the all-twos configuration}

Let $c$ be a configuration on a sandpile graph $\Gamma$, not necessarily an element of $\mathcal{S}(\Gamma)$, the sandpile group. If $k$ is a nonnegative integer, let $k \cdot c$ denote the vertex-wise addition of $c$ with itself $k$ times, without stabilizing. The order of $c$, denoted $\operatorname{order}(c)$, is the smallest positive integer $k$ such that $k \cdot c$ is in the image of the reduced Laplacian of $\Gamma$. If $c$ is recurrent, then the order of $c$ is the same as its order as an element of $\mathcal{S}(\Gamma)$ according to the isomorphism (2).

Consider the sandpile grid graph, $\mathrm{S} \Gamma_{m \times n}$, with $m, n \geqslant 2$. For each nonnegative integer $k$, let $\vec{k}_{m \times n}=k \cdot \overrightarrow{1}_{m \times n}$ be the all-ks configuration on $\mathrm{S} \Gamma_{m \times n}$ consisting of $k$ grains of sand on each vertex. The motivating question for this section is: what is the order of $\overrightarrow{1}_{m \times n}$ ? Since $\overrightarrow{1}_{m \times n}$ has up-down and left-right symmetry, its order must divide the order of the group of symmetric recurrents on $\mathrm{S} \Gamma_{m \times n}$ calculated in Theorems 15, 18, and 23. The number of domino tilings of a $2 n \times 2 n$ checkerboard can be written as $2^{n} a_{n}^{2}$ where $a_{n}$ is an odd integer (cf. Proposition 27). Our main result is Theorem 29 which, through Corollary 30, says that the order of $\overrightarrow{2}_{2 n \times 2 n}$ divides $a_{n}$.

Proposition 25. Let $m, n \geqslant 2$.

1. The configuration $\overrightarrow{1}_{m \times n}$ is not recurrent.

2. The configuration $\overrightarrow{2}_{m \times n}$ is recurrent.

3. The order of $\overrightarrow{1}_{m \times n}$ is either $\operatorname{order}\left(\overrightarrow{2}_{m \times n}\right)$ or $2 \operatorname{order}\left(\overrightarrow{2}_{m \times n}\right)$.

4. Let $\widetilde{\Delta}_{m \times n}$ be the reduced Laplacian of $\mathrm{S} \Gamma_{m \times n}$. The order of $\overrightarrow{1}_{m \times n}$ is the smallest integer $k$ such that $k \cdot \widetilde{\Delta}_{m \times n}^{-1} \overrightarrow{1}_{m \times n}$ is an integer vector.

Proof. Parts (1) and (2) follow from the burning algorithm (Theorem 1). 
For part (3), let $\alpha=\operatorname{order}\left(\overrightarrow{1}_{m \times n}\right)$ and $\beta=\operatorname{order}\left(\overrightarrow{2}_{m \times n}\right)$, and let $e$ be the identity of $\mathcal{S}\left(\mathrm{S} \Gamma_{m \times n}\right)$. Let $\widetilde{\mathcal{L}}$ denote the image of the reduced Laplacian, $\widetilde{\Delta}$, of $\mathrm{S} \Gamma_{m \times n}$. Since $e=\left(2 \alpha \cdot \overrightarrow{1}_{m \times n}\right)^{\circ}=\left(\alpha \cdot \overrightarrow{2}_{m \times n}\right)^{\circ}$ and $e=\left(\beta \cdot \overrightarrow{2}_{m \times n}\right)^{\circ}=\left(2 \beta \cdot \overrightarrow{1}_{m \times n}\right)^{\circ}$, we have

$$
2 \beta \geqslant \alpha \geqslant \beta \text {. }
$$

We have $(2 \beta-\alpha) \cdot \overrightarrow{1}_{m \times n}=0 \bmod \widetilde{\mathcal{L}}$. Suppose $\alpha \neq 2 \beta$. It cannot be that $2 \beta-\alpha=1$. Otherwise, $\overrightarrow{1}_{m \times n}=0 \bmod \widetilde{\mathcal{L}}$. It would then follow that $\overrightarrow{2}_{m \times n}$ and $\overrightarrow{3}_{m \times n}$ are recurrent elements equivalent to 0 modulo $\widetilde{\mathcal{L}}$, whence, $\overrightarrow{2}_{m \times n}=\overrightarrow{3}_{m \times n}=e$, a contradiction. Thus, $(2 \beta-\alpha) \cdot \overrightarrow{1}_{m \times n} \geqslant \overrightarrow{2}_{m \times n}$. Since $\overrightarrow{2}_{m \times n}$ is recurrent, $\left((2 \beta-\alpha) \cdot \overrightarrow{1}_{m \times n}\right)^{\circ}$ is recurrent and equivalent to 0 modulo $\widetilde{\mathcal{L}}$, and thus must be the $e$. So $2 \beta-\alpha \geqslant \alpha$, and the right side of (15) implies $\alpha=\beta$, as required.

Now consider part (4). The order of $\overrightarrow{1}_{m \times n}$ is the smallest positive integer $k$ such that $k \cdot \overrightarrow{1}_{m \times n}=0 \bmod \widetilde{\mathcal{L}}$, i.e., for which there exists an integer vector $v$ such that $k \cdot \overrightarrow{1}_{m \times n}=$ $\widetilde{\Delta}_{m \times n} v$. The result follows.

Example 26. We have order $\left(\overrightarrow{1}_{2 \times 2}\right)=2 \operatorname{order}\left(\overrightarrow{2}_{2 \times 2}\right)=2$, and $\operatorname{order}\left(\overrightarrow{1}_{2 \times 3}\right)=\operatorname{order}\left(\overrightarrow{2}_{2 \times 3}\right)=$ 7. In general, we do not know which case will hold in part 3 of Proposition 25.

Table 1 records the order of $\overrightarrow{2}_{m \times n}$ for $m, n \in\{2,3, \ldots, 10\}$. Perhaps the most striking

\begin{tabular}{c|lllllllll}
$m \backslash n$ & 2 & 3 & 4 & 5 & 6 & 7 & 8 & 9 & 10 \\
\hline 2 & 1 & 7 & 5 & 9 & 13 & 47 & 17 & 123 & 89 \\
3 & $\cdot$ & 8 & 71 & 679 & 769 & 3713 & 8449 & 81767 & 93127 \\
4 &. &. & 3 & 77 & 281 & 4271 & 2245 & 8569 & 18061 \\
5 &. &. &. & 52 & 17753 & 726433 & 33507 & 24852386 & 20721019 \\
6 &. &. &. &. & 29 & 434657 & 167089 & 265721 & 4213133 \\
7 &. &. &. &. &. & 272 & 46069729 & 8118481057 & 4974089647 \\
8 &. &. &. &. &. &. & 901 & 190818387 & 1031151241 \\
9 &. &. &. &. &. &. &. & 73124 & 1234496016491 \\
10 &. &. &. &. &. &. &. &. & 89893
\end{tabular}

Table 1: Order of the all-2s element on $\mathrm{S}_{m \times n}$ (symmetric in $m$ and $n$ ).

feature of Table 1 is the relatively small size of the numbers along the diagonal $(m=n)$. It seems natural to group these according to parity. The sequence $\left\{\overrightarrow{2}_{2 n \times 2 n}\right\}_{n \geqslant 1}$ starts $1,3,29,901,89893, \ldots$, which is the beginning of the famous sequence, $\left(a_{n}\right)_{n \geqslant 1}$, we now describe. The following was established independently by several people (cf. [19]):

Proposition 27. The number of domino tilings of a $2 n \times 2 n$ checkerboard has the form

$$
2^{n} a_{n}^{2}
$$

where $a_{n}$ is an odd integer. 
For each positive integer $n$, let $P_{n}$ be the sandpile graph with vertices

$$
V\left(P_{n}\right)=\left\{v_{i, j}: 1 \leqslant i \leqslant n \text { and } 1 \leqslant j \leqslant i\right\} \cup\{s\} .
$$

Each $v_{i, j}$ is connected to those vertices $v_{i^{\prime}, j^{\prime}}$ such that $\left|i-i^{\prime}\right|+\left|j-j^{\prime}\right|=1$. In addition, every vertex of the form $v_{i, n}$ is connected to the sink vertex, $s$. The first few cases are illustrated in Figure 19. Next define a family of triangular checkerboards, $H_{n}$, as in Figure 20. The

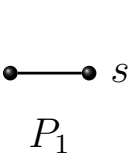

$P_{1}$

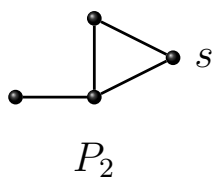

Figure 19:

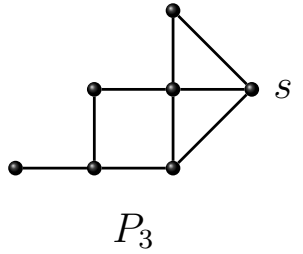

$P_{3}$

checkerboard $H_{n}$ for $n \geqslant 2$ is formed by adding a $2 \times(2 n-1)$ array (width-by-height) of squares to the right of $H_{n-1}$. These graphs were introduced by M. Ciucu [6] and later
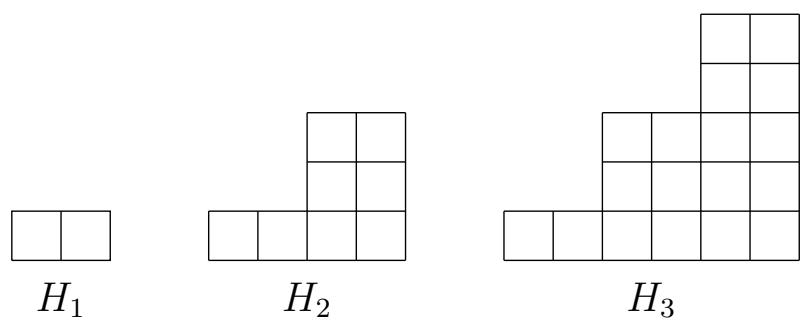

Figure 20:

used by L. Pachter [35] to give the first combinatorial proof of Proposition 27. As part of his proof, Pachter shows that $a_{n}$ is the number of domino tilings of $H_{n}$.

As noted in [21], considering $H_{n}$ as a planar graph and taking its dual (forgetting about the unbounded face of $H_{n}$ ) gives the graph $\mathcal{H}\left(P_{n}\right)$ corresponding to $P_{n}$ under the generalized Temperley bijection of Section 3. See Figure 21.

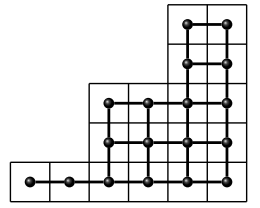

Figure 21: $H_{3}$ and $\mathcal{H}\left(P_{3}\right)$.

Proposition 28. The number of elements in the sandpile group for $P_{n}$ is

$$
\# \mathcal{S}\left(P_{n}\right)=a_{n}
$$

where $a_{n}$ is as in Proposition 27. 
Proof. The number of domino tilings of $H_{n}$ equals the number of perfect matchings of $\mathcal{H}\left(P_{n}\right)$. By the generalized Temperley bijection, the latter is the number of spanning trees of $P_{n}$, and hence, the order of the sandpile group of $P_{n}$. As mentioned above, Pachter shows in [35] that $a_{n}$ is the number of domino tilings of $H_{n}$.

The main result of this section is the following:

Theorem 29. Let $\left\langle\overrightarrow{2}_{2 n \times 2 n}\right\rangle$ be the cyclic subgroup of $\mathcal{S}\left(\mathrm{S}_{2 n \times 2 n}\right)$ generated by the all- $2 \mathrm{~s}$ element of $\Gamma_{2 n \times 2 n}$, and let $\overrightarrow{2}_{n}$ denote the all-2s element on $P_{n}$. Then the mapping

$$
\psi:\left\langle\overrightarrow{2}_{2 n \times 2 n}\right\rangle \rightarrow \mathcal{S}\left(P_{n}\right),
$$

determined by $\psi\left(\overrightarrow{2}_{2 n \times 2 n}\right)=\overrightarrow{2}_{n}$, is a well-defined injection of groups.

Proof. Let $\widetilde{V}_{n}$ and $\widetilde{V}_{2 n \times 2 n}$ denote the non-sink vertices of $P_{n}$ and $\mathrm{S} \Gamma_{2 n \times 2 n}$, respectively. We view configurations on $P_{n}$ as triangular arrays of natural numbers and configurations on $\mathrm{S}_{2 n \times 2 n}$ as $2 n \times 2 n$ square arrays of natural numbers. Divide the $2 n \times 2 n$ grid by drawing bisecting horizontal, vertical, and diagonal lines, creating eight wedges. Define $\phi: \mathbb{Z} \widetilde{V}_{n} \rightarrow \mathbb{Z} \widetilde{V}_{2 n \times 2 n}$, by placing a triangular array in the position of one of these wedges, then flipping about lines, creating a configuration on $\mathrm{S}_{2 n \times 2 n}$ with dihedral symmetry. Figure 22 illustrates the case $n=4$.

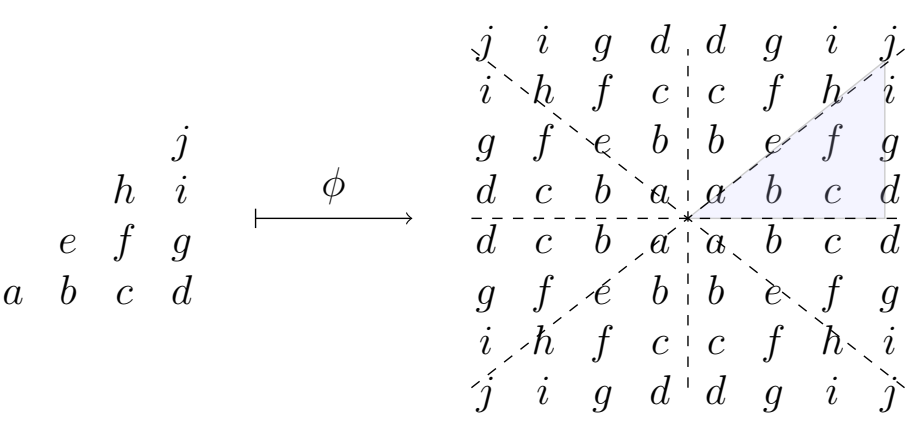

Figure 22: $\phi: \mathbb{Z} P_{4} \rightarrow \mathbb{Z S} \Gamma_{8 \times 8}$.

We define special types of configurations on $P_{n}$. First, let $s_{n}$ be the configuration in which the number of grains of sand on each vertex records that vertex's distance to the sink; then let $t_{n}$ denote the sandpile with no sand except for one grain on each vertex along the boundary diagonal, i.e., those vertices with degree less than 3. Figure 23 illustrates the case $n=4$.

Let $\widetilde{\Delta}_{n}$ and $\widetilde{\Delta}_{2 n \times 2 n}$ be the reduced Laplacians for $P_{n}$ and $\mathrm{S} \Gamma_{2 n \times 2 n}$, respectively. The following are straightforward calculations:

1. $\widetilde{\Delta}_{n} s_{n}=t_{n}$.

2. If $c \in \mathbb{Z} P_{n}$, then $\widetilde{\Delta}_{2 n \times 2 n}(\phi(c))$ equals $\phi\left(\widetilde{\Delta}_{n}(c)\right)$ at all non-sink vertices of $\mathrm{S}_{2 n \times 2 n}$ except along the diagonal and anti-diagonal, where they differ by a factor of 2 :

$$
\widetilde{\Delta}_{2 n \times 2 n}(\phi(c))_{i j}= \begin{cases}2 \phi\left(\widetilde{\Delta}_{n}(c)\right)_{i j} & \text { for } i=j \text { or } i+j=2 n+1, \\ \phi\left(\widetilde{\Delta}_{n}(c)\right)_{i j} & \text { otherwise. }\end{cases}
$$




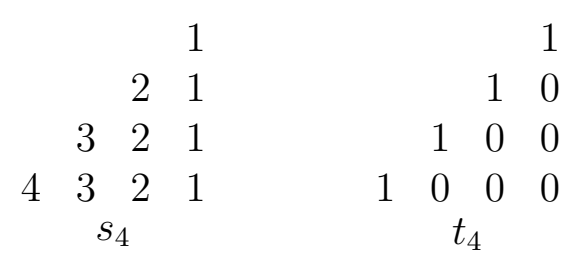

Figure 23: Special configurations on $P_{4}$.

Let $\widetilde{\mathcal{L}}_{n} \subset \mathbb{Z} V_{n}$ and $\widetilde{\mathcal{L}}_{2 n \times 2 n} \subset \mathbb{Z} V_{2 n \times 2 n}$ denote the images of $\widetilde{\Delta}_{n}$ and $\widetilde{\Delta}_{2 n \times 2 n}$, respectively. Identify the sandpile groups of $P_{n}$ and $\mathrm{S} \Gamma_{2 n \times 2 n}$ with $\mathbb{Z} V_{n} / \widetilde{\mathcal{L}}_{n}$ and $\mathbb{Z} V_{2 n \times 2 n} / \widetilde{\mathcal{L}}_{2 n \times 2 n}$, respectively. To show that $\psi$ is well-defined and injective, we need to show that $k \overrightarrow{2}_{n} \in \widetilde{\mathcal{L}}_{n}$ for some integer $k$ if and only if $k \overrightarrow{2}_{2 n \times 2 n} \in \widetilde{\mathcal{L}}_{2 n \times 2 n}$. Since the reduced Laplacians are invertible over $\mathbb{Q}$, there exist unique vectors $x$ and $y$ defined over the rationals such that

$$
\widetilde{\Delta}_{n} x=\overrightarrow{2}_{n} \text { and } \quad \widetilde{\Delta}_{2 n \times 2 n} y=\overrightarrow{2}_{2 n \times 2 n} .
$$

Using the special configurations $s_{n}$ and $t_{n}$ and the two calculations noted above,

$$
\widetilde{\Delta}_{n} x=\overrightarrow{2}_{n} \quad \Longrightarrow \quad \widetilde{\Delta}_{n}\left(x-s_{n}\right)=\overrightarrow{2}_{n}-t_{n} \quad \Longrightarrow \quad \widetilde{\Delta}_{2 n \times 2 n} \phi\left(x-s_{n}\right)=\overrightarrow{2}_{2 n \times 2 n} .
$$

In other words,

$$
y=\phi\left(x-s_{n}\right) .
$$

Using the fact that $\widetilde{\Delta}_{n}$ is invertible over $\mathbb{Q}$, we see that $k \overrightarrow{2}_{n} \in \widetilde{\mathcal{L}}_{n}$ if and only if $k x$ has integer coordinates. By (16), this is the same as saying $k y$ has integer components, which in turn is equivalent to $k \overrightarrow{2}_{2 n \times 2 n} \in \widetilde{\mathcal{L}}_{2 n \times 2 n}$, as required.

Combining this result with Proposition 28 gives

Corollary 30. The order of $\overrightarrow{2}_{2 n \times 2 n}$ divides $a_{n}$.

\section{Conclusion}

We conclude with a list of suggestions for further work.

1. Theorem 18 states that the number of domino tilings of a Möbius checkerboard equals the number of domino tilings of an associated ordinary checkerboard after assigning weights to certain domino positions. We would like to see a direct bijective proof - one that does not rely on the Lu-Wu formula (and thus giving a new proof of that formula). For instance, consider the tiling of the $4 \times 4$ checkerboard that appears second in the top row of Figure 8. It has one domino of weight 2. So this weighted tiling should correspond to two tilings of the $4 \times 4$ Möbius checkerboard. Presumably, one of these two tilings is just the unweighted version of the given tiling. One might imagine that the other tiling would result from pushing the single blue domino to the right one square so that it now wraps around on the Möbius checkerboard, and then making room for this displacement by systematically shifting the other dominos. 
2. Section 5 is motivated by Irena Swanson's question: what is the order of the all-1s configuration, $\overrightarrow{1}_{m \times n}$, on the $m \times n$ sandpile grid graph? Proposition 25 (3) shows this order is either the same as or twice the order of the all- $2 \mathrm{~s}$ configuration, $\overrightarrow{2}_{m \times n}$. It would be nice to know when each case holds. Corollary 30 says the order of $\overrightarrow{2}_{2 n \times 2 n}$ divides the integer $a_{n}$ of Proposition 27, connected with domino tilings. When is this order equal to $a_{n}$ ? Ultimately, of course, we would like to know the answer to Swanson's original question.

3. Example 16 introduces an action of the sandpile group of the $2 m \times 2 n$ sandpile grid graph on the domino tilings of the $2 m \times 2 n$ checkerboard. Perhaps this group action deserves further study.

4. To summarize some of the main ideas of this paper, suppose a group acts on an arbitrary sandpile graph $\Gamma$. If the corresponding symmetrized reduced Laplacian or its transpose is the (ordinary) reduced Laplacian of a sandpile graph $\Gamma^{\prime}$, then Proposition 10 yields a group isomorphism between the symmetric configurations on $\Gamma$ and the sandpile group $\mathcal{S}\left(\Gamma^{\prime}\right)$ of $\Gamma^{\prime}$. By the matrix-tree theorem, the size of the latter group is the number of spanning trees of $\Gamma^{\prime}$ (and, in fact, as mentioned earlier, $\mathcal{S} \Gamma^{\prime}$ is well-known to act freely and transitively on the set of spanning trees of $\Gamma^{\prime}$ ). The generalized Temperley bijection then gives a correspondence between the spanning trees of $\Gamma^{\prime}$ and perfect matchings of a corresponding graph, $\mathcal{H}\left(\Gamma^{\prime}\right)$. Thus, the number of symmetric recurrents on $\Gamma$ equals the number of perfect matchings of $\mathcal{H}\left(\Gamma^{\prime}\right)$. We have applied this idea to the case of a particular group acting on sandpile grid graphs. Does it lead to anything interesting when applied to other classes of graphs with group action? The Bachelor's thesis of the first author [15] includes a discussion of the case of a dihedral action on sandpile grid graphs.

\section{Acknowledgments}

We thank Irena Swanson for providing initial motivation. We thank the organizers of the Special Session on Laplacian Growth at the Joint Mathematics Meeting, New Orleans, LA, 2011 at which some of this work was presented, and we thank Lionel Levine, in particular, for encouragement and helpful remarks. We would like to acknowledge the mathematical software Sage [44] and the Online Encyclopedia of Integer Sequences [33] which were both essential for our investigations. Finally, we are appreciative of comments received from an anonymous referee.

\section{References}

[1] Per Bak, Chao Tang, and Kurt Wiesenfeld, Self-Organized Criticality: An Explanation of $1 / f$ Noise, Phys. Rev. Lett. 59 (1987), no. 4, 381-384.

[2] Matthew Baker and Serguei Norine, Riemann-Roch and Abel-Jacobi theory on a finite graph, Adv. Math. 215 (2007), no. 2, 766-788.

[3] Matthew Baker and Farbod Shokrieh, Chip-firing games, potential theory on graphs, and spanning trees, J. Combin. Theory Ser. A 120 (2013), no. 1, 164-182. 
[4] N. L. Biggs, Chip-firing and the critical group of a graph, J. Algebraic Combin. 9 (1999), no. 1, 25-45.

[5] Norman Biggs, Algebraic potential theory on graphs, Bull. London Math. Soc. 29 (1997), no. 6, 641-682.

[6] Mihai Ciucu, Weigen Yan, and Fuji Zhang, The number of spanning trees of plane graphs with reflective symmetry, J. Combin. Theory Ser. A 112 (2005), no. 1, 105116.

[7] F. Cools, J. Draisma, S. Payne, and E. Robeva, A tropical proof of the Brill-Noether Theorem, Adv. Math. 230 (2012), 759-776.

[8] Robert Cori and Dominique Rossin, On the sandpile group of dual graphs, European J. Combin. 21 (2000), no. 4, 447-459.

[9] Deepak Dhar, Self-organized critical state of sandpile automaton models, Phys. Rev. Lett. 64 (1990), no. 14, 1613-1616.

[10] _ Theoretical studies of self-organized criticality, Phys. A 369 (2006), no. 1, $29-70$.

[11] Anton Dochtermann and Raman Sanyal, Laplacian ideals, arrangements, and resolutions, Preprint, to appear in J. Algebraic Combin. arXiv:1212.6244

[12] Natalie J. Durgin, Abelian Sandpile Model on Symmetric Graphs, Bachelor's thesis, Harvey Mudd College, 2009.

[13] Anne Fey, Lionel Levine, and Yuval Peres, Growth rates and explosions in sandpiles, J. Stat. Phys. 138 (2010), no. 1-3, 143-159.

[14] Anne Fey, Lionel Levine, and David B. Wilson, Approach to criticality in sandpiles, Phys. Rev. E (3) 82 (2010), no. 3, 031121, 14.

[15] Laura Florescu, New connections between the Abelian Sandpile Model and domino tilings, Bachelor's thesis, Reed College, 2011.

[16] Alexander E. Holroyd, Lionel Levine, Karola Mészáros, Yuval Peres, James Propp, and David B. Wilson, Chip-firing and rotor-routing on directed graphs, In and out of equilibrium. 2, Progr. Probab., vol. 60, Birkhäuser, Basel, 2008, pp. 331-364.

[17] Sam Hopkins and David Perkinson, Bigraphical Arrangements, Preprint, to appear in Trans. Amer. Math. Soc. arXiv:1212.4398

[18] _ Orientations, semiorders, arrangements, and parking functions, Elec. J. of Combin. 19 (2012), no. 4.

[19] P. John, H. Sachs, and H. Zernitz, Problem 5. Domino Covers in Square Chessboards, Zastosowania Matematyki (Applicationes Mathematicae) XIX (1987), no. 3-4, 635641.

[20] P. W. Kasteleyn, The statistics of dimers on a lattice, I. the number of dimer arrangements on a quadratic lattice, Physica 27 (1961), 1209-1225.

[21] Richard W. Kenyon, James G. Propp, and David B. Wilson, Trees and matchings, Electron. J. Combin. 7 (2000), Research Paper 25, 34 pp. (electronic). 
[22] Yvan Le Borgne and Dominique Rossin, On the identity of the sandpile group, Discrete Math. 256 (2002), no. 3, 775-790, LaCIM 2000 Conference on Combinatorics, Computer Science and Applications (Montreal, QC).

[23] Lionel Levine and Yuval Peres, Strong spherical asymptotics for rotor-router aggregation and the divisible sandpile, Potential Anal. 30 (2009), no. 1, 1-27.

[24] Dino J. Lorenzini, A finite group attached to the Laplacian of a graph, Discrete Math. 91 (1991), no. 3, 277-282.

[25] Wentao T. Lu and F. Y. Wu, Close-packed dimers on nonorientable surfaces, Phys. Lett. A 293 (2002), no. 5-6, 235-246.

[26] Madhusudan Manjunath, Frakn-Olaf Schreyer, and John Wilmes, Minimal Free Resolutions of the g-Parking Function Ideal and the Toppling Ideal, Preprint, to appear in Trans. Amer. Math. Soc. arXiv:1210.7569

[27] Madhusudan Manjunath and Bernd Sturmfels, Monomials, binomials and RiemannRoch, J. Algebraic Combin. 37 (2013), no. 4, 737-756.

[28] Sergei Maslov, Sandpile applet, Online: accessed May 22, 2014. http://www.cmth. bnl.gov/ maslov/Sandpile.htm

[29] J. C. Mason and D. C. Handscomb, Chebyshev polynomials, Chapman \& Hall/CRC, Boca Raton, FL, 2003.

[30] Criel Merino López, Chip firing and the Tutte polynomial, Ann. Comb. 1 (1997), no. 3, 253-259.

[31] Luca-Guido Molinari, Determinants of block tridiagonal matrices, Linear Algebra and its Applications 429 (2008), 2221-2226.

[32] Gregg Musiker, The critical groups of a family of graphs and elliptic curves over finite fields, J. Algebraic Combin. 30 (2009), no. 2, 255-276.

[33] The On-Line Encyclopedia of Integer Sequences, A067502, oeis.org/A081567.

[34] S. Ostojic, Patterns formed by addition of grains to only one site of an abelian sandpile., Phys. A 318 (2003), 187-199.

[35] Lior Pachter, Combinatorial approaches and conjectures for 2-divisibility problems concerning domino tilings of polyominoes, Electron. J. Combin. 4 (1997), no. 1, Research Paper 29, 10 pp. (electronic).

[36] Gugliemo Paoletti, Deterministic Abelian Sandpile Models and Patterns, Ph.D. thesis, Univeristà di Pisa, 2012.

[37] Wesley Pedgen and Charles K Smart, Apollonian structure in the Abelian Sandpile, Preprint, 2012. arXiv:1208.4839

[38] Wesley Pegden and Charles K. Smart, Convergence of the Abelian sandpile, Duke Math. J. 162 (2013), no. 4, 627-642.

[39] David Perkinson, Jacob Perlman, and John Wilmes, Primer for the algebraic geometry of sandpiles, Tropical and Non-Archimedean Geometry, Contemp. Math., vol. 605, Amer. Math. Soc., Providence, RI, 2013, pp. 211-256. 
[40] Alexander Postnikov and Boris Shapiro, Trees, parking functions, syzygies, and deformations of monomial ideals, Trans. Amer. Math. Soc. 356 (2004), no. 8, 3109-3142 (electronic).

[41] V. B. Priezzhev, Structure of two-dimensional sandpile: I. Height probabilities, J. Stat. Phys. 74 (1994), no. 5-6, 955-979.

[42] Tridib Sadhu and Deepak Dhar, Pattern formation in growing sandpiles with multiple sources or sinks, J. Stat. Phys. 138 (2010), no. 4-5, 815-837.

[43] Eugene Speer, Asymmetric abelian sandpile models, J. Stat. Phys. (1993), 61-74.

[44] W. A. Stein et al., Sage Mathematics Software (Version 5.1), The Sage Development Team, 2012. http://www. sagemath.org

[45] H. N. V. Temperley and Michael E. Fisher, Dimer problem in statistical mechanicsan exact result, Philos. Mag. (8) 6 (1961), 1061-1063.

[46] Wikipedia, Chebyshev polynomials - Wikipedia, the free encyclopedia, 2012. Online; accessed May 22, 2014. http://en.wikipedia.org/wiki/Chebyshev_polynomials 